\title{
Article \\ Effect of Aging and Moisture Damage on Fatigue Cracking Properties in Asphalt Mixtures
}

\author{
Sung Lin Yang ${ }^{1}$, Cheolmin Baek ${ }^{2}$ and Hee Beom Park ${ }^{2, *(\mathbb{D}}$ \\ 1 Department of Civil Engineering, Kyung Hee University, Yongin-si 17104, Korea; onionlyn77@daum.net \\ 2 Department of Highway\& Transportation Research, Korea Institute of Civil Engineering and Building \\ Technology, Goyang-si 10223, Korea; cmbaek@kict.re.kr \\ * Correspondence: heebeompark@kict.re.kr; Tel.: +82-31-910-0627
}

Citation: Yang, S.L.; Baek, C.; Park, H.B. Effect of Aging and Moisture Damage on Fatigue Cracking Properties in Asphalt Mixtures. Appl. Sci. 2021, 11, 10543. https://doi.org/10.3390/ app112210543

Academic Editor: Luís Picado Santos

Received: 15 October 2021

Accepted: 8 November 2021

Published: 9 November 2021

Publisher's Note: MDPI stays neutral with regard to jurisdictional claims in published maps and institutional affiliations.

Copyright: (c) 2021 by the authors. Licensee MDPI, Basel, Switzerland. This article is an open access article distributed under the terms and conditions of the Creative Commons Attribution (CC BY) license (https:// creativecommons.org/licenses/by/ $4.0 /)$.
Abstract: Recently, damage to asphalt pavements in South Korea has increased because of direct and indirect factors caused by abnormal climatic changes, such as torrential rains, prolonged heatwaves in summer, and heavy snowfall in winter. Additionally, the use of medium and heavy vehicles is also a contributing factor. Therefore, an experimental procedure to study the moisture damage and fracture properties of asphalt pavements considering the recent changes in precipitation properties, deterioration of road pavement, and traffic conditions in South Korea was developed in this study. Additionally, changes in material properties according to the indoor aging process and fatigue crack resistance were evaluated for hot mix and warm mix asphalt mixtures of the same grade and different initial production and construction temperatures. To evaluate the effect of aging and moisture damage on fatigue cracking, the experimental coefficient values of the predictive model were calculated for each condition. It was observed that the material properties of hot mix asphalt mixtures changed with an increase in aging and moisture damage. The service life of the pavement was reduced by approximately $40-80 \%$ owing to moisture damage, whereas aging had a greater effect on fatigue life as the service life increased.

Keywords: asphalt pavement; moisture damage; fracture properties; aging; hot mix asphalt (HMA); warm mix asphalt (WMA)

\section{Introduction}

The moisture damage phenomenon that occurs in asphalt pavements is defined as the loss of rigidity and durability due to adhesion and viscosity between the asphalt film. The aggregate of the asphalt mixture is weakened because of the penetration of moisture by precipitation [1]. The moisture damage of asphalt pavement is associated with various common properties, such as bleeding, cracking, plastic deformation, labeling, and potholes. Additionally, it is affected by various factors, such as hydrophilicity of the aggregate, traffic volume and traffic load, pavement design, and construction [2].

The mechanism of moisture damage in asphalt pavement is very complex, and research to identify the factors causing moisture damage is still in progress by various researchers. Various indoor test methods have been developed to simulate the properties of asphalt mixtures for moisture damage. In addition, the effect of aging and also cracking study of asphaltic mixtures and bituminous materials has also been investigated in recent years [3-7]. According to Brown et al. (2000), the tensile strength ratio (TSR) of the asphalt mixture before and after moisture damage prescribed in AASHTO T 283 is the most effective method for understanding the sensitivity of asphalt mixture to moisture damage, which is a representative and simple test method generally applied natively and in other countries [8]. TSR specified in AASHTO T 283 is an evaluation method that compares only the tensile strength of the asphalt mixture before and after moisture damage [9]. Therefore, it cannot be used to determine the mechanical properties of the asphalt mixture and the 
direct cause of moisture damage based on the test results, thus limiting the prediction of the service life of pavement owing to moisture damage [10].

In general, the aging of asphalt pavement occurs because of various factors, and the stiffness of the asphalt pavement gradually increases with aging. However, the increased stiffness is inevitably vulnerable to moisture. Sarsam and AL-Zubaidi (2016) reported that the stiffness of asphalt mixtures with short-term aging (STA) and long-term aging (LTA) increased in the range of $7-54 \%$, and its fatigue life reduced in the range of $70-87 \%$ owing to moisture damage [11]. The brittleness of asphalt binder increased with aging, viscous properties of asphalt deteriorated with increased stiffness, and fatigue life decreased owing to the loss of adhesion and viscosity between the aggregate and asphalt binder. Prabir Kumar Das et al. (2015) developed a fracture energy simulation model using various aging and moisture damage methods to study the effects of aging of asphalt mixture and moisture on the service life of asphalt pavement. It was observed that the moisture damage was accelerated by the loss of adhesion and viscosity of the asphalt binder due to aging [12]. Lee et al. (2017) predicted the pavement lifespan at the design stage by measuring the dynamic modulus of the asphalt mixture, which is a material property used for mechanical-empirical pavement design before and after moisture damage and reported that moisture damage reduced the fatigue life of the pavement resulting in plastic deformation [10].

Recently, the damage of asphalt pavements in South Korea has increased because of direct and indirect factors caused by abnormal climatic changes, such as torrential rains, prolonged heatwaves in summer, and heavy snowfall in winter, along with the effects of medium and heavy vehicles. Recent pothole damage on national roads increased by more than 1.5 times from 25,231 cases in 2009 to 38,382 cases in 2012. In particular, the number of potholes in Seoul, which is the capital of South Korea, increased by 2.6 times in one year from approximately 30,000 in 2009 to 78,000 in 2010 [13]. Figure 1 shows, for Seoul, the amount of precipitation and the number of days that saw precipitation, compared to the number of potholes over the past 10 years (2010-2020) [14]. It demonstrates that the number of potholes is more closely correlated to the number of days that saw precipitation than to the average annual precipitation. In other words, more rainy days are likelier to result in greater numbers of potholes.

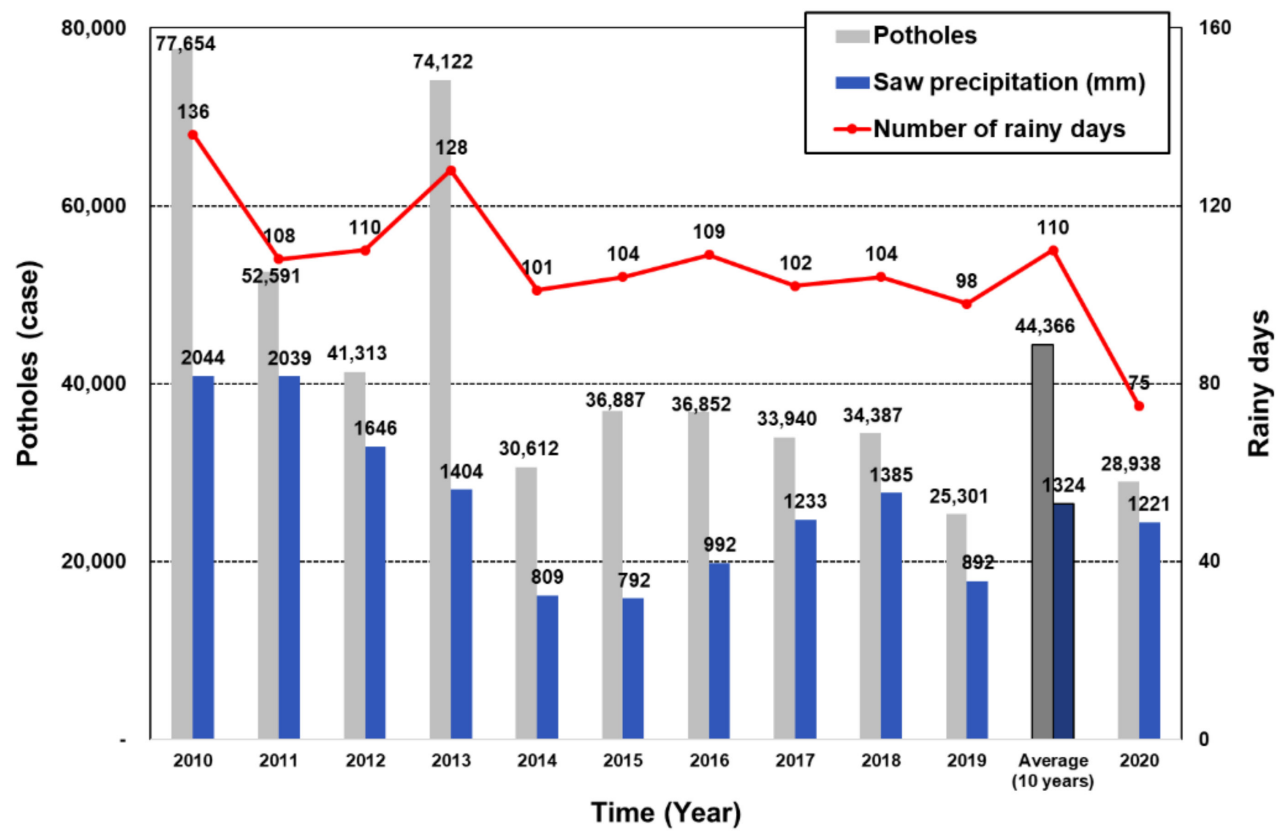

Figure 1. The amount of precipitation and the number of days that saw precipitation compared to the number of potholes [14]. 
In this study, an experimental procedure to study the moisture damage and damage properties of asphalt pavements considering the recent precipitation properties, deterioration of road pavement, and changes in traffic conditions in South Korea was developed. The aim of this study is to evaluate the effect and contribution of moisture damage properties and fatigue cracking according to the degree of aging in asphalt pavements.

An evaluation of the effect on the material properties of the aged asphalt mixture due to moisture damage was evaluated with a focus on fatigue cracks, which is a form of asphalt pavement failure, at room temperature. Furthermore, to understand the effect of moisture damage of asphalt mixture due to aging on the material and fatigue cracking properties, dynamic modulus and direct tensile cyclic load tests were performed on hot mix asphalt (HMA) and warm mix asphalt (WMA) mixtures generally used in South Korea.

\section{Materials and Mix Design}

\subsection{Materials}

In this study, the main specifications of the used asphalt binder for manufacturing HMA and WMA has penetration levels of 70-80 and PG 64-22 produced by company A. Table 1 shows the basic properties of asphalt binders of HMA and WMA.

Table 1. Test variables and designation of specimens.

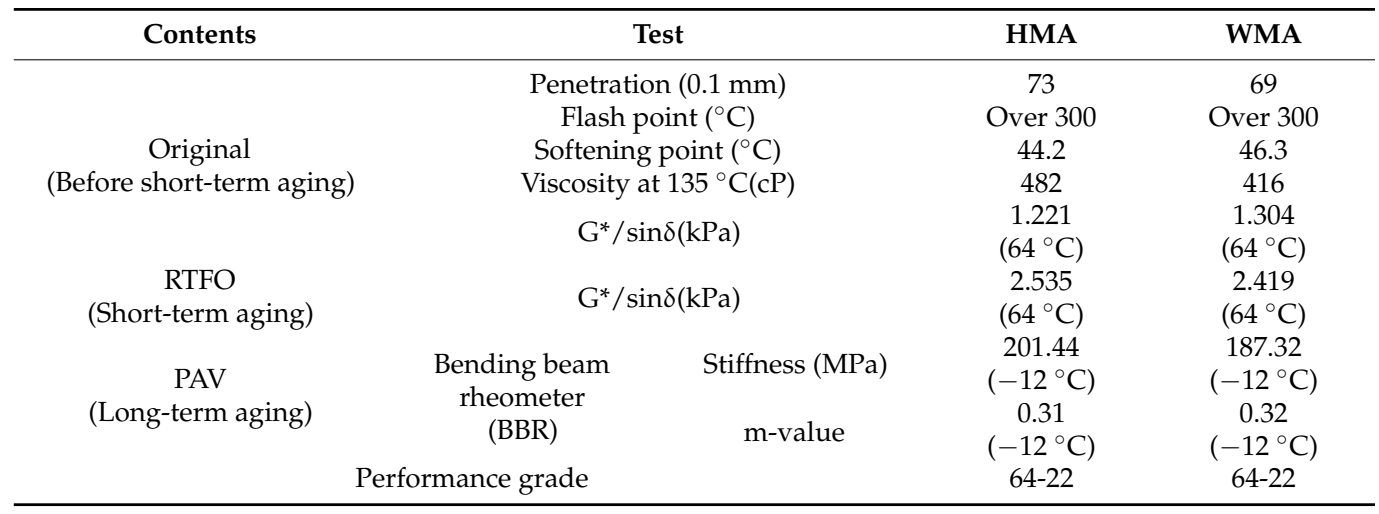

The WMA additive used in this study was prepared using the Fischer-Tropsch synthesis method based on a polyethylene-based wax. It is characterized by the effects of improving the fluidity of the asphalt binder and reducing the manufacturing temperature and compaction temperature of the asphalt mixture. The asphalt binder used in HMA and WMA mixtures consists of asphalt for road paving with a penetration standard of $70-80$ and a PG grade of $64-22$. Similarly, the aggregate consists of crushed granite aggregate satisfying the WC-3 (nominal maximum size $19 \mathrm{~mm}$ ) particle size standard presented in the "Asphalt Mixture Production and Construction Guidelines (2017)" by the Ministry of Land, Infrastructure, and Transport [15]. In addition, the thick aggregate used was a single-particle first-class aggregate, and an aggregate with a knitting stone rate of less than $10 \%$ of the thick aggregates was used. Tables 2 and 3 show the results of the quality test of the aggregation

Table 2. Basic properties of the used aggregates.

\begin{tabular}{ccccc}
\hline \multirow{2}{*}{ Contents } & \multirow{2}{*}{ Spec. } & \multicolumn{3}{c}{ Test Result } \\
\cline { 3 - 5 } & & Coarse Agg. & Fine Agg. & Filler \\
\hline Specific gravity & $\geq 2.5$ & 2.89 & 2.77 & 2.68 \\
Water absorption $(\%)$ & $\leq 3.0$ & 0.33 & 0.65 & - \\
LA abrasion coefficient $(\%)$ & $\leq 35$ & 12.2 & - & - \\
\hline
\end{tabular}


Table 3. Flat and elongation of the used aggregates.

\begin{tabular}{ccc}
\hline The Size of Agg. & FE Rate (\%, of Weight) & Specification (\%) \\
\hline $20 \mathrm{~mm}$ & 4.8 & $\leq$ Under \\
$13 \mathrm{~mm}$ & 7.6 & $\leq$ Under \\
\hline
\end{tabular}

\subsection{Mix Design}

The objective of the mix design of asphalt mixture is to determine the optimal asphalt content based on the combination of materials used and ensure resistance to plastic deformation and fatigue cracking. In this study, one type of aggregate and two types of asphalt were used to design the mix using the Marshall mix design method suggested by the Ministry of Land, Infrastructure, and Transport. Relevant tests were conducted on HMA and WMA mixtures under similar conditions. The optimum asphalt contents were applied to the mix design for HMA and WMA mixtures having the same particle size. The compaction temperature was $140 \pm 3^{\circ} \mathrm{C}$ for the HMA mixture and $115 \pm 3^{\circ} \mathrm{C}$ for the WMA mixture. Table 4 shows the results of the mix design of the asphalt mixtures used in this study.

Table 4. Test variables and designation of specimens.

\begin{tabular}{|c|c|c|c|c|c|c|c|}
\hline $\begin{array}{ll}\text { Division } & \text { Item } \\
\end{array}$ & $\begin{array}{l}\text { Asphalt Content } \\
(\%)\end{array}$ & Air Void (\%) & Density $\left(\mathrm{g} / \mathrm{cm}^{3}\right)$ & VMA (\%) & VFA (\%) & Stability (kgf) & Flow Value $(0.1 \mathrm{~mm})$ \\
\hline Hot mix asphalt (HMA) binder & 5.1 & 4.0 & 2.377 & 15.58 & 74.2 & 1488 & 31 \\
\hline Warm mix asphalt (WMA) binder & 5.1 & 4.1 & 2.361 & 15.33 & 73.7 & 1662 & 34 \\
\hline
\end{tabular}

\subsection{Fabrication of Specimens}

In this study, specimens were prepared for indoor experiments for the evaluation of aging and moisture damage in HMA and WMA mixtures. Based on the mix design specifications shown in Table 4, the superpave gyratory compactor (SGC) was used to ensure the same air void.

To evaluate the material properties according to aging and moisture damage, a test specimen was prepared by selecting the target air void as $7.0 \% \pm 0.5 \%$ according to the AASHTO T 283 standard for the air void of the specimen. The final target air void refers to the air void of the specimen after coring and cutting after specimen fabrication. To produce a specimen with the target air void, the amount of asphalt mixture was determined based on the correlation between the amount of asphalt mixture and air void. Figure 2 shows the correlation between the amount of asphalt mixture and air void. A specimen is prepared by selecting the air void with respect to the amount of mixture determined based on the given correlation.

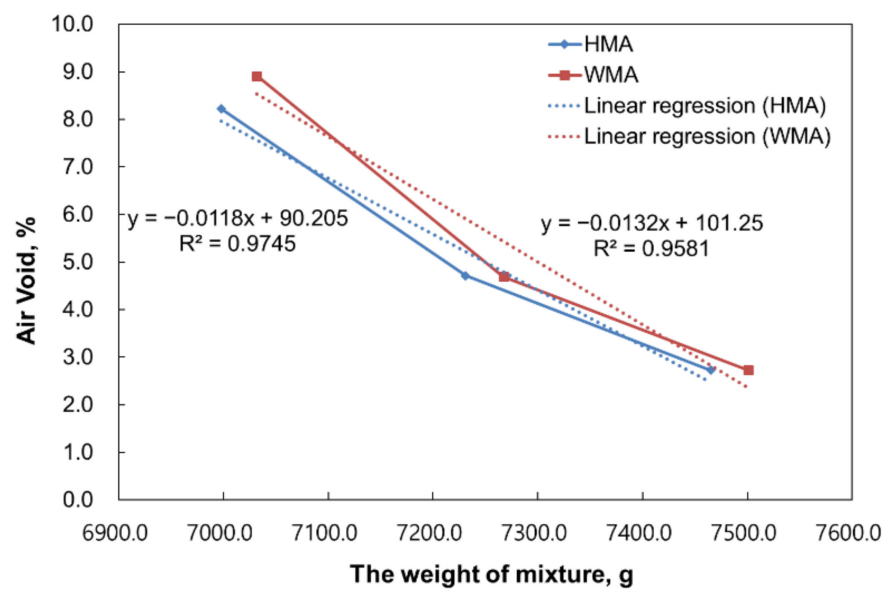

Figure 2. Correlation between the amount of asphalt mixture and air void. 
The specimen used for the test is cylindrical in shape with a diameter of $100 \mathrm{~mm}$ and a height of $150 \mathrm{~mm}$. In "E*-Dynamic Modulus Test Protocol (2003)", a specimen with a size of 6.7 inches and a diameter of 6 inches was manufactured using the SGC to dynamic modulus test of the asphalt mixture presented in the AASHTO 2002 Guide [16]. After curing is completed, a procedure for performing cutting and coring on the manufactured specimen again with a height of $150 \mathrm{~mm}$ and a diameter of $100 \mathrm{~mm}$ was presented. In this study, the air void of the specimen cut and coated in accordance with the method was selected to be $7 \% \pm 0.5 \%$, and the specimen was manufactured in the same. To ensure the same air void as shown in Figure 3a, the specimen is fabricated with a diameter of $150 \mathrm{~mm}$ and height of $178 \mathrm{~mm}$, followed by coring and cutting as shown in Figure 3b.

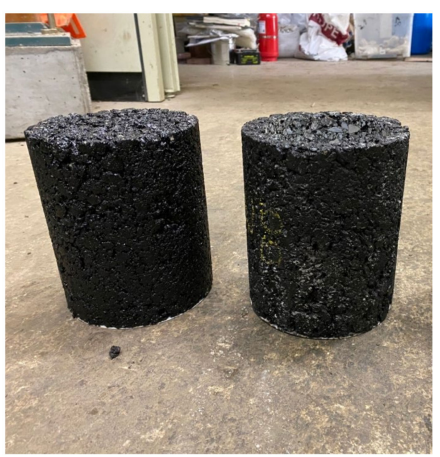

(a)

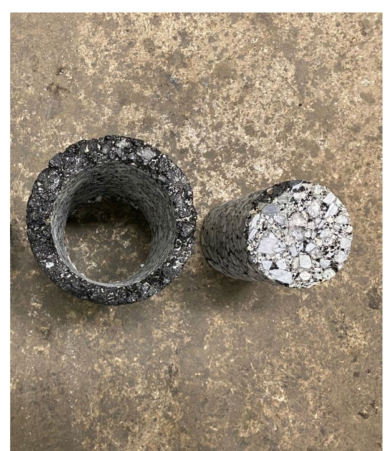

(b)

Figure 3. Fabrication of specimens: (a) primary specimens and (b) final specimens.

\section{Experimental Program}

To evaluate the changes in physical properties and resistance to fatigue cracking in the aged asphalt mixture according to the degree of aging and moisture damage, an experiment was conducted. The experimental procedure is depicted in the form of a flowchart, as shown in Figure 4.

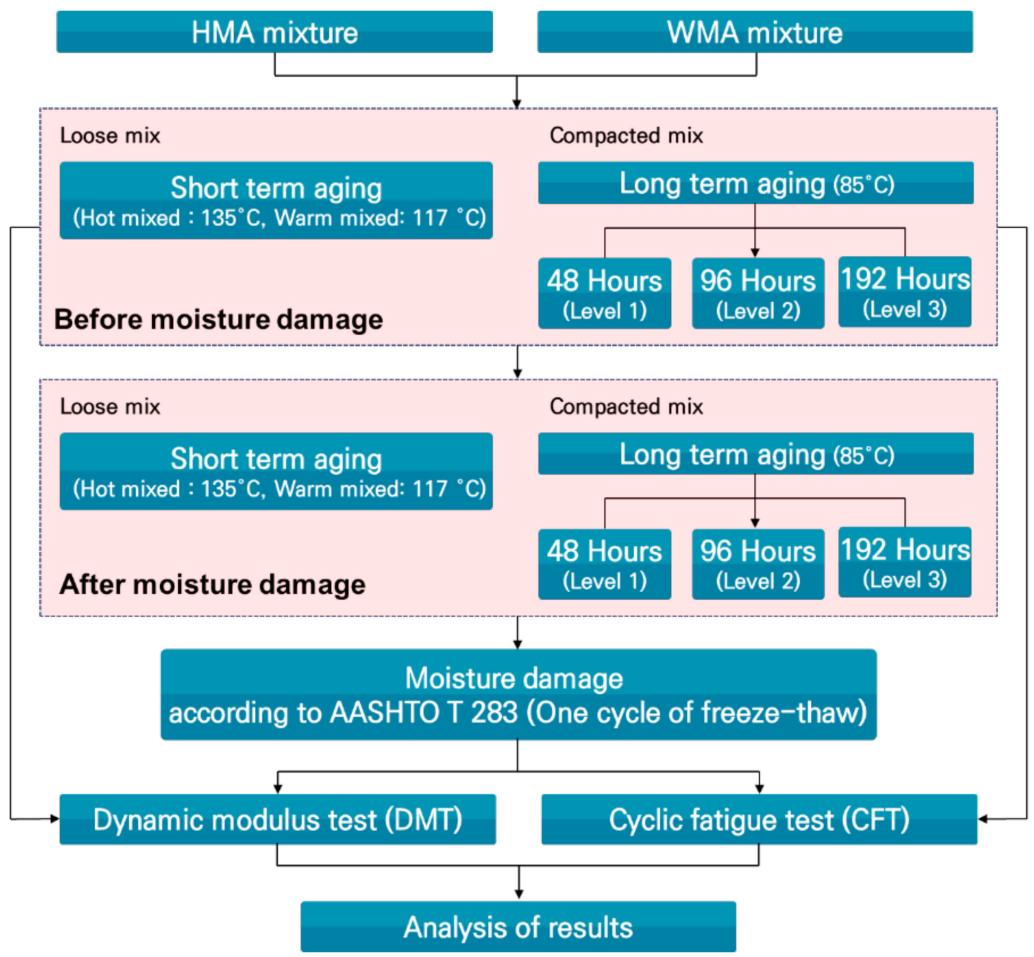

Figure 4. Flowchart of the experiment. 
Baek (2010) developed a model to determine the resistance of the asphalt mixture to top-down cracking according to its aging phase [17]. To determine the damage caused by the degree of aging of the asphalt mixture, the test specimen was aged according to the method proposed by SHRP, and short-term aging is considered 1 to 2 years, long-term aging $48 \mathrm{~h}$ is considered 4 years, $96 \mathrm{~h}$ is 7.5 years, and $192 \mathrm{~h}$ is 18 years [18]. In this study, long-term aging time was also selected as 48, 96, and $192 \mathrm{~h}$ with reference to the above-mentioned studies.

\subsection{Aging Method}

In NCHRP 09-54, two major methods were proposed for simulating aging during the co-use of an asphalt mixture. The first is an aging method based on the shape of the material, and the second is a method based on the pressure level (heat and pressure aging) [19]. In this study, the asphalt mixture for each material type was aged by heating to simulate aging during the use of asphalt pavement. Indoor aging was performed in a heating oven, and STA was performed according to AASHTO R 30 [20]. According to NCHRP 9-43, STA was performed at $135{ }^{\circ} \mathrm{C}$ for four hours for the HMA mixture and at $117^{\circ} \mathrm{C}$ for two hours for the WMA mixture [21]. For LTA, each asphalt mixture that undergoes STA was compacted with an SGC to produce a specimen. After coring and cutting, the samples were placed in a heating oven at $85^{\circ} \mathrm{C}$ and aged for $48 \mathrm{~h}$ (LTA 1), $96 \mathrm{~h}$ (LTA 2), and $192 \mathrm{~h}$ (LTA 3). Figure 5 shows the state of the specimen after STA and LTA 3 after LTA.

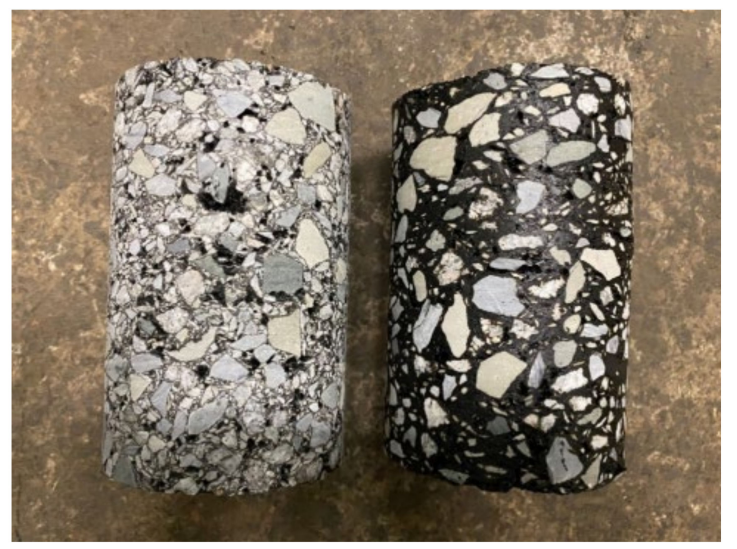

Figure 5. Specimen after short-term aging (left) and specimen after long-term aging (right).

\subsection{Moisture Damage Method}

In this study, the immersion method implemented in the modified Ratman test method of AASHTO T 283 was applied to simulate the effect of moisture during the use of asphalt pavement and moisture damage of the asphalt mixture [9]. Accordingly, a cycle of moisture freeze-thaw was applied to simulate the moisture damage caused by repeated moisture effects. After moisture damage, the moisture inside the specimen was forcibly removed using the CoreLok equipment for the performance test. To return to the same conditions as before moisture damage, the specimens were placed in a dryer at $25 \pm 1^{\circ} \mathrm{C}$ for $24 \mathrm{~h}$ after removing moisture and before the test.

\subsection{Dynamic Modulus Test}

Asphalt mixtures in the linear viscoelastic range are thermorheologically simple (TRS), which can be expressed as a reduced time or reduced frequency by combining the effects of time and temperature. The reduced frequency is calculated using the time-temperature shift factor $\left(a_{T}\right)$ as shown in Equation (1).

$$
f_{R}=f \times \alpha_{T}
$$


This allows the overall graph to be shifted horizontally, creating a single curve (master curve) to describe the behavior of the asphalt mixture at a significantly reduced frequency. The time-temperature shift factor represents the horizontal shift on a logarithmic scale required to build the master curve. The amount of shift is determined by the change in reference temperature.

The master curve is fitted to the form of a sigmoidal function as in Equation (2), and the relationship between shift factor and temperature can be expressed as a quadratic function. The solver provided by the EXCEL program was used to determine the sigmoidal coefficient and time-temperature shift factor by selecting a value that minimized the logarithmic error for the equation and measured value. At a specific temperature, the value of the transfer coefficient becomes zero and the temperature at this time is the reference temperature that can be determined at any experimental temperature; however, an intermediate value is generally used. This logarithmic shift factor is fitted by Equation (3) and is used to determine the elastic modulus at any temperature and frequency using Equation (2) in combination with the coefficient determined in Equation (3).

$$
\begin{aligned}
& \log \left|E^{*}\right|=\alpha+\frac{b}{1+\frac{1}{e^{d+g^{*} \log \left(f_{R}\right)}}} \\
& \log \left(\alpha_{T}\right)=\alpha_{1} T^{2}+\alpha_{2} T+\alpha_{3}
\end{aligned}
$$

In this study, the dynamic modulus was determined using the stress control method in uniaxial compression mode. Experiments were carried out at 20, 10, 5, 1, 0.5, and $0.1 \mathrm{~Hz}$ load cycles at temperatures of $-5,5,20,40$, and $54{ }^{\circ} \mathrm{C}$ according to the method proposed by AASHTO TP 62 as shown in Figure 6 [22]. For the dynamic modulus test, the EN Standard equipment by IPC was used, and a total of three extensometers (length $70 \mathrm{~mm}$ ) were installed at intervals of $120^{\circ}$ in the center of the specimen.

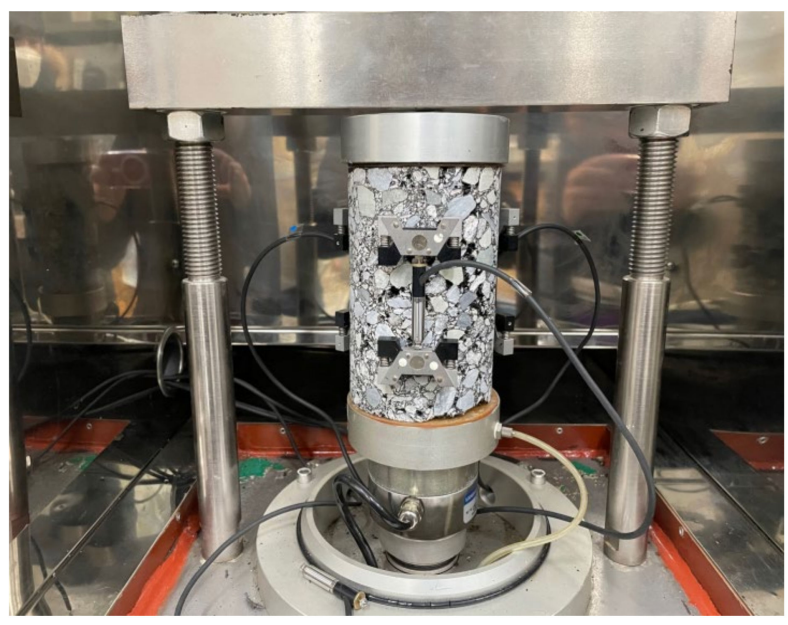

Figure 6. Dynamic modulus test.

\subsection{Fatigue Cracking Resistance Test}

To evaluate the resistance to fatigue cracking, a cyclic fatigue test was performed using two experimental methods: load control and displacement control. In this study, a controlled crosshead cyclic loading (CX) test was conducted. This allows the simulation of the load conditions that occur during actual use by applying a mixed type of load (neither stress nor strain control) on the specimen. The controlled CX test was performed by loading at $20{ }^{\circ} \mathrm{C}$ at a rate of $10 \mathrm{~Hz}$. The fracture time was determined as the time point of the phase angle exhibiting a rapid change using the method proposed by Reese (1997) [23]. Figure 7 shows the changes in the dynamic modulus and phase angle according to the number of loading cycles. In general, as the number of loading cycles increases, the dynamic modulus gradually decreases, and the phase angle increases. As shown in Figure 7, the phase angle 
decreases rapidly after a load is applied for a certain period of time, which indicates that the specimen is broken.

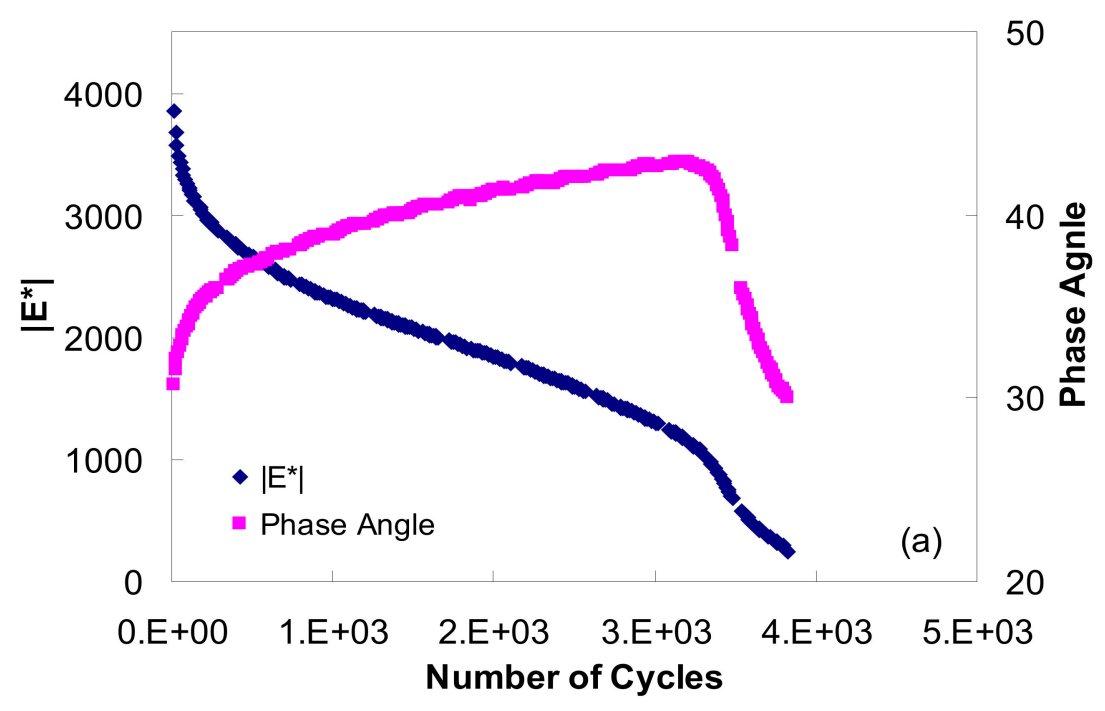

Figure 7. Curves depicting the changes in dynamic modulus and phase angles according to loading [23].

In the fatigue test performed previously, the dynamic modulus itself does not directly reflect the fatigue performance owing to aging and moisture damage. In other words, the fatigue performance should be evaluated considering the resistance to deformation, resistance to damage, and fracture properties. The fatigue test reflects the fatigue properties of the material; however, fatigue life or cracking in the asphalt pavement should be predicted based on the traffic load and environmental conditions. Therefore, to use the fatigue performance prediction equation presented by the Mechanistic-Empirical Pavement Design Guide in the United States, this study intends to obtain the coefficient values for each condition (for each stage of aging and before/after moisture damage) required for the fatigue performance prediction equation based on the results of the indoor experiment. The allowable fatigue cracking cycles under each condition were determined using the coefficient values obtained in this study.

$$
N_{f}=C k_{1}\left(\frac{1}{\varepsilon_{t}}\right)^{k_{2}}\left(\frac{1}{E}\right)^{k_{3}}
$$

where $N_{f}$ is the number of repetitions to fatigue cracking; $\varepsilon_{t}$ is the tensile strain at the critical location; $E$ denotes the stiffness of the material; $k_{1}, k_{2}$, and $k_{3}$ are the laboratory regression coefficients; and $C$ is the laboratory-to-field adjustment factor.

\section{Test Results}

\subsection{Dynamic Modulus Test Results}

The dynamic modulus master curve was plotted for a reference temperature of $5{ }^{\circ} \mathrm{C}$ in semi-log and log-log scale graphs. The semi-log scale clearly indicates the change in elastic modulus in the low-temperature region, and the log-log scale clearly indicates the change in elastic modulus in the high-temperature region. As the temperature increases, the phase angle master curve first increases and then decreases after a certain temperature. At low temperatures (high loading cycle, high frequency), elastic properties of the asphalt binder are dominant rather than the interlocking of the aggregates. As the temperature increases (low loading cycle, low frequency), elastic properties of the asphalt binder gradually decrease while the elastic properties increase owing to the interlocking between the aggregates resulting in a decrease in the phase angle. In this study, a dynamic modulus 
master curve was prepared using the FlexMAT program presented by the Federal Highway Administration (FHWA).

Figures 8 and 9 show the dynamic modulus test results for the HMA and WMA mixtures for each stage of aging. The dynamic modulus increases with aging in both HMA and WMA mixtures. The increase is higher in the WMA mixture than in the HMA mixture because the STA method applied to both the mixtures was different. Thus, the dynamic modulus after STA was higher in the HMA mixture than in the WMA mixture. Even with aging, the elastic modulus of the HMA mixture was consistently higher. The effect of early aging (STA) seemed to have a lasting effect on the WMA mixture compared to the HMA mixture. The dynamic modulus of the HMA mixture increased by approximately $10-35 \%$ based on STA at $10 \mathrm{~Hz}$. As aging progressed, the modulus of elasticity increased by approximately $50 \%$ with an increase in the temperature or decrease in the loading cycle. However, the modulus of elasticity increased by approximately $20-60 \%$ in the WMA mixture at $10 \mathrm{~Hz}$ as a result of STA. With an increase in the temperature or a decrease in the loading cycle, the modulus of elasticity increased by approximately $100 \%$ or more, indicating that the WMA mixture was more sensitive to aging than the HMA mixture. Thus, the aging effect was accelerated as the initial aging was lower than that of the HMA mixture. This was consistent with the results of the study by Safaei et al. (2014), who reported a trend in the difference between HMA and WMA due to early aging, which gradually decreased as aging progressed [24].

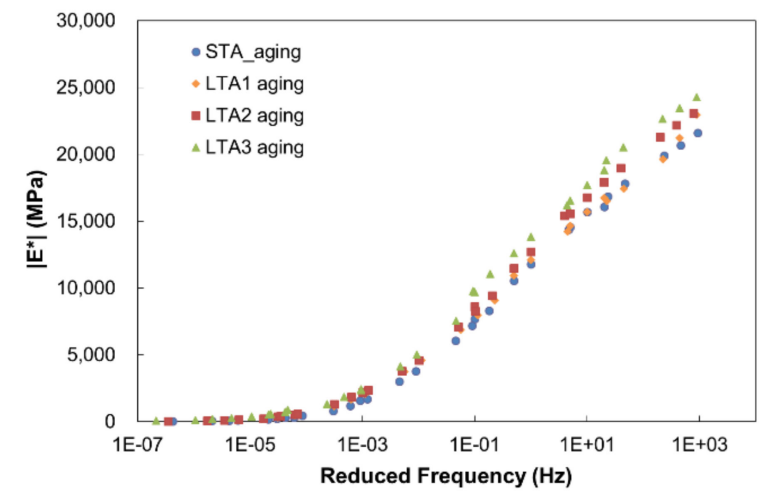

(a)

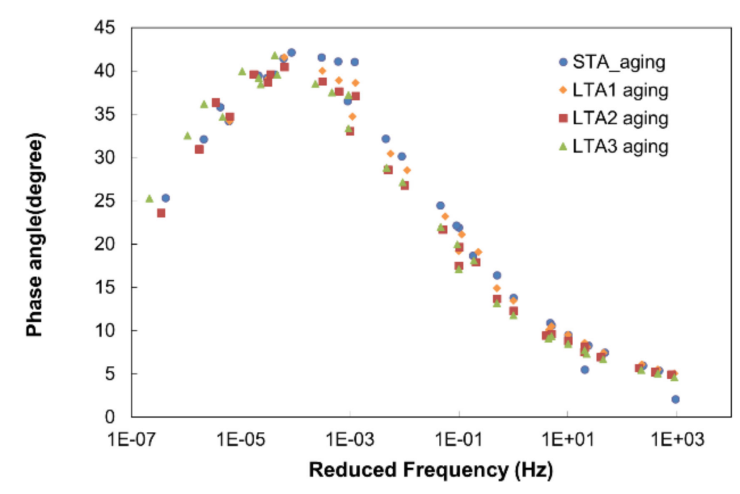

(c)

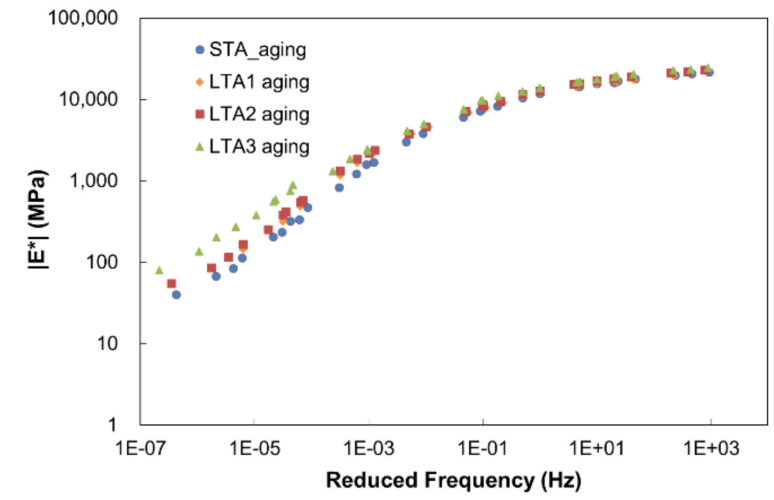

(b)

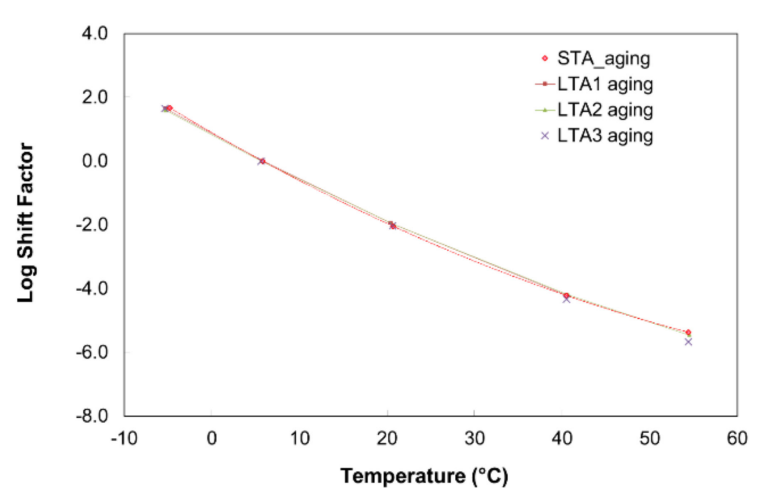

(d)

Figure 8. Dynamic modulus test results of HMA for each stage of aging: (a) dynamic modulus master curve (semi-log scale), (b) dynamic modulus master curve (log-log scale), (c) phase angle master curve, and (d) shift factor. 


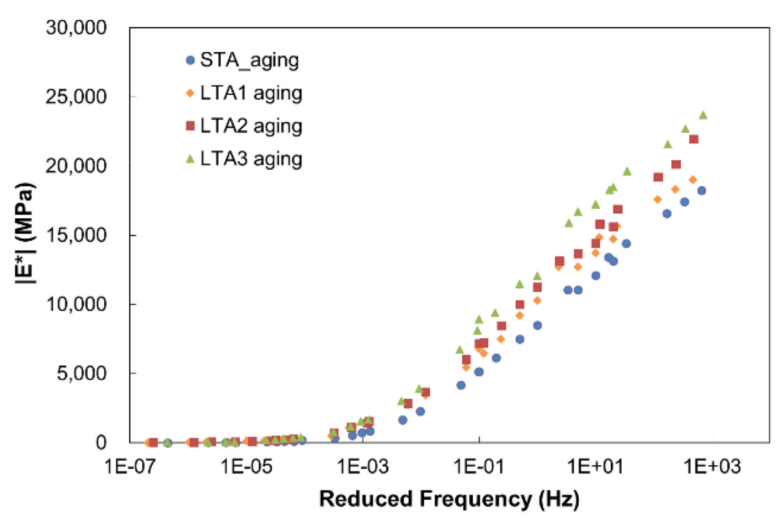

(a)

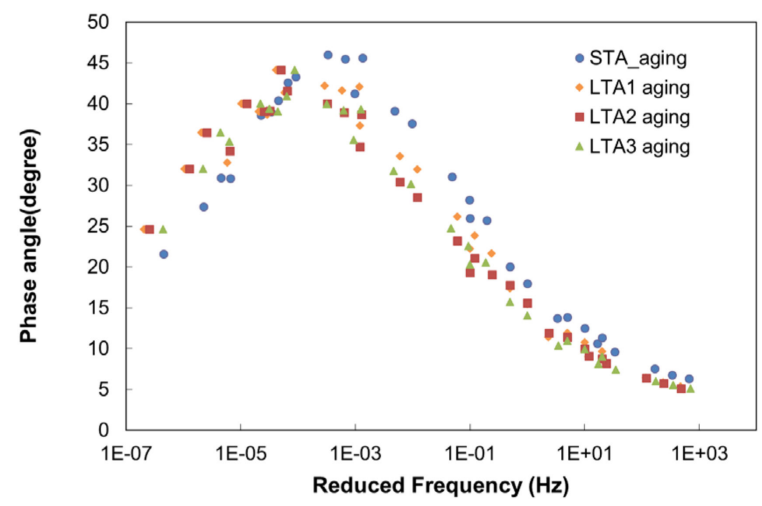

(c)

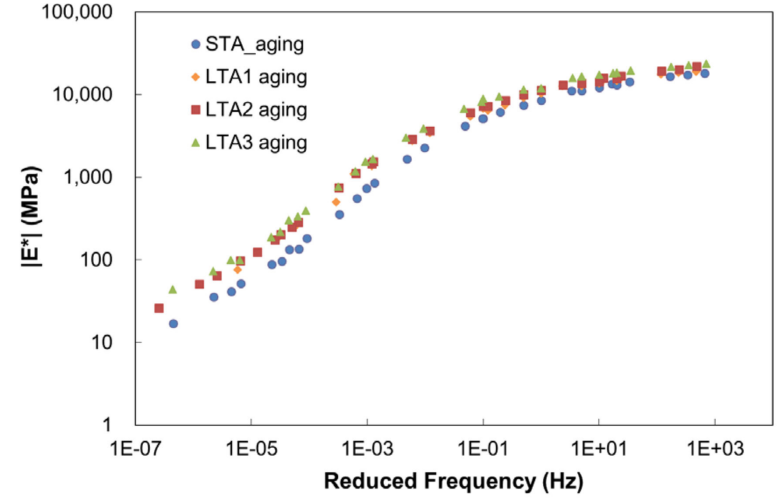

(b)

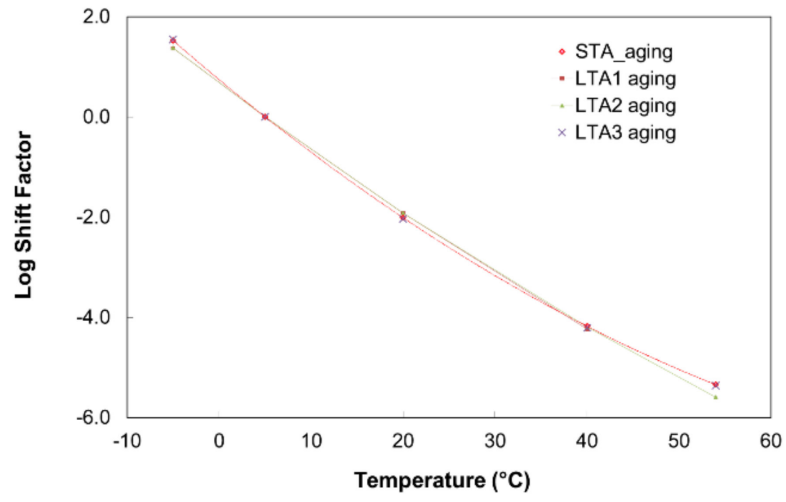

(d)

Figure 9. Dynamic modulus test results of WMA for each stage of aging: (a) dynamic modulus master curve (semi-log scale), (b) dynamic modulus master curve (log-log scale), (c) phase angle master curve, and (d) shift factor.

Figures 10 and 11 show the results of the dynamic modulus test conducted on HMA and WMA mixtures after moisture damage for each stage of aging. After moisture damage, the dynamic modulus of the HMA mixture increased by approximately $15-42 \%$ at $20^{\circ} \mathrm{C}$ and $10 \mathrm{~Hz}$; however, the size of the overall dynamic modulus was smaller than that before moisture damage. In addition, the increase in the rate of the dynamic modulus after moisture damage on the WMA mixture was approximately $50-100 \%$, indicating that the change in physical properties was larger than that before moisture damage. The WMA mixture also exhibited a lower overall dynamic modulus after the moisture damage than that before moisture damage. In both HMA and WMA mixtures, the change in material properties after moisture damage was greater than that before moisture damage. After moisture damage, the dynamic modulus increased with aging; however, the dynamic modulus after moisture damage was lower than that before moisture damage, suggesting that moisture damage reduced the performance of the material. 


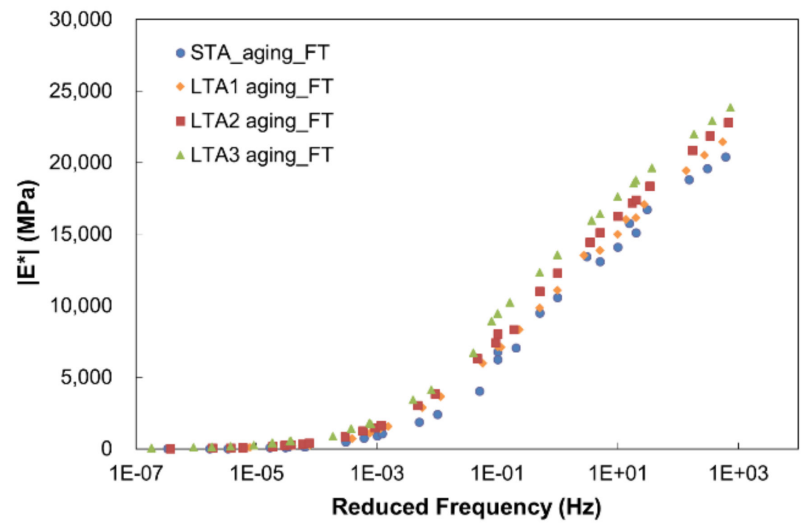

(a)

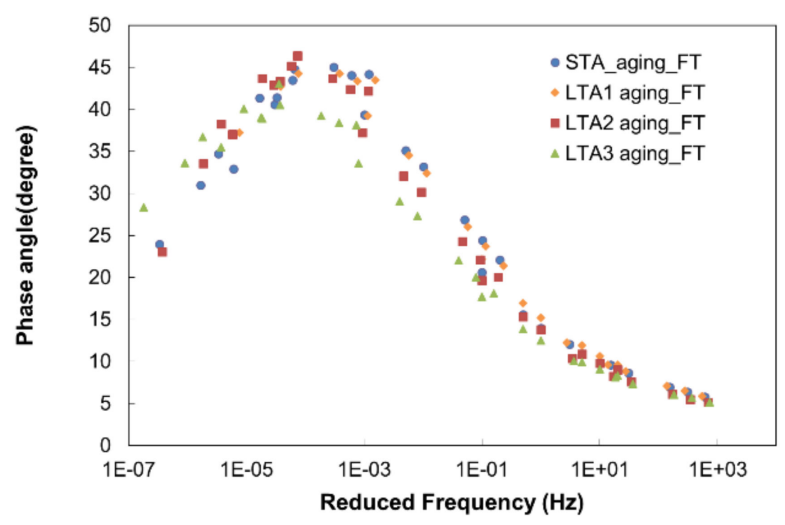

(c)

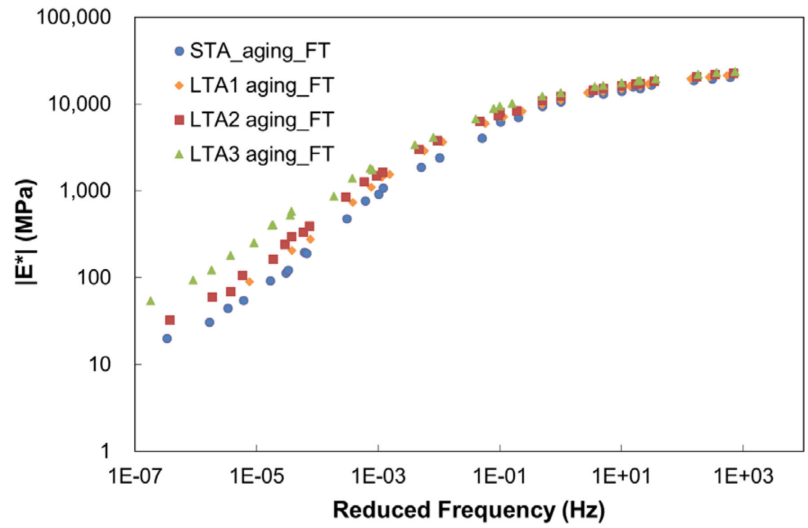

(b)

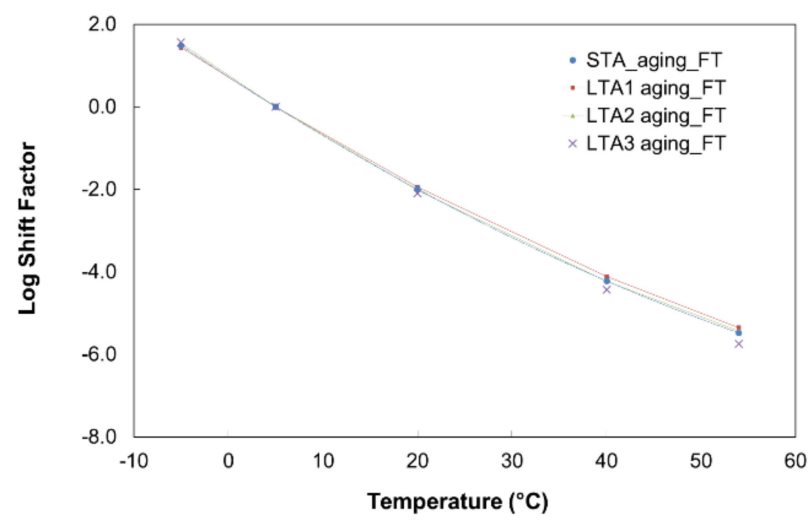

(d)

Figure 10. Dynamic modulus test results of HMA after moisture damage for each stage of aging: (a) dynamic modulus master curve (semi-log scale), (b) dynamic modulus master curve (log-log scale), (c) phase angle master curve, and (d) shift factor.

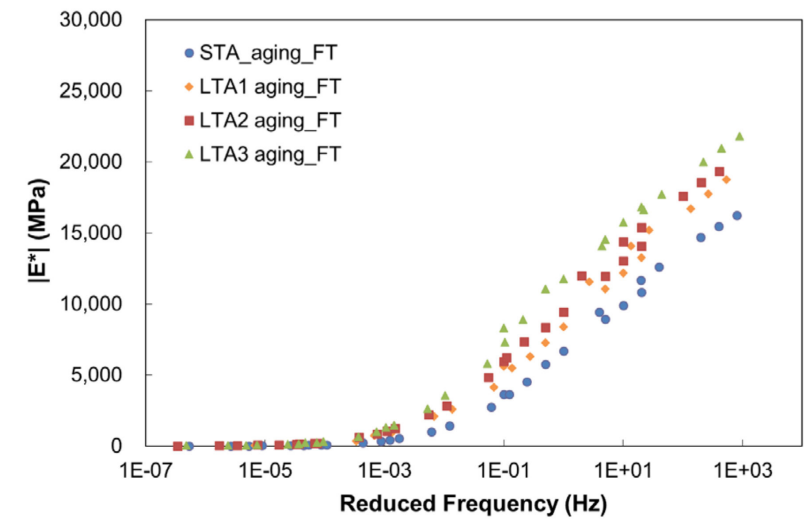

(a)

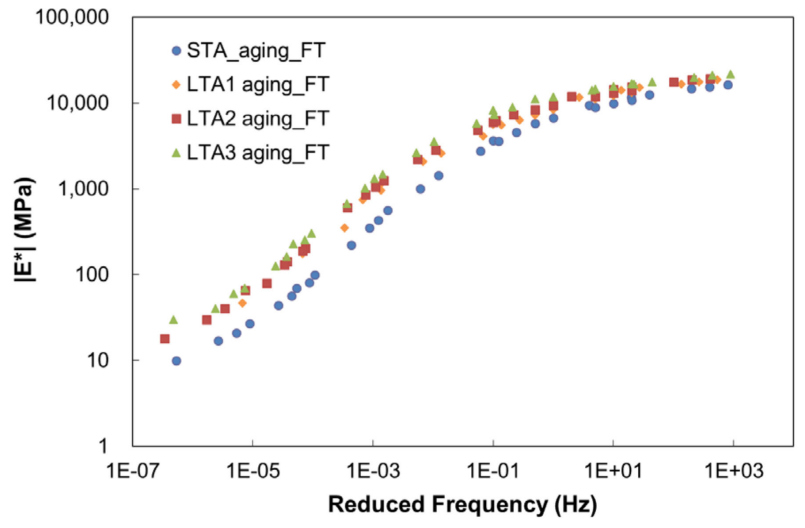

(b)

Figure 11. Cont. 


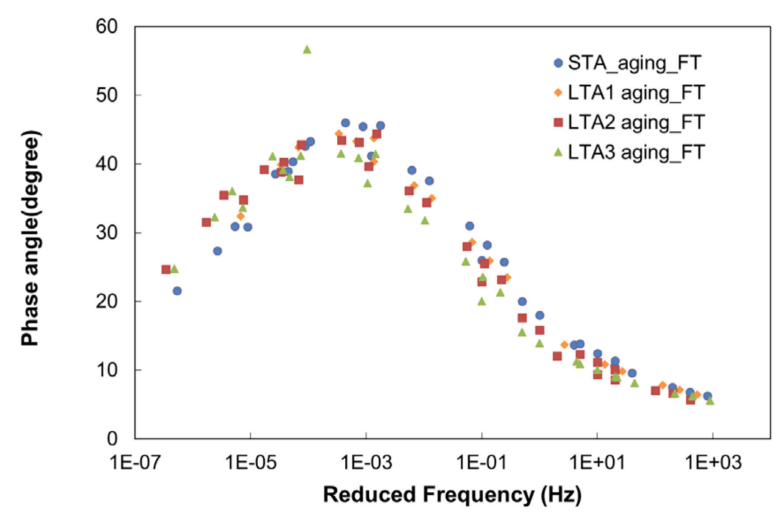

(c)

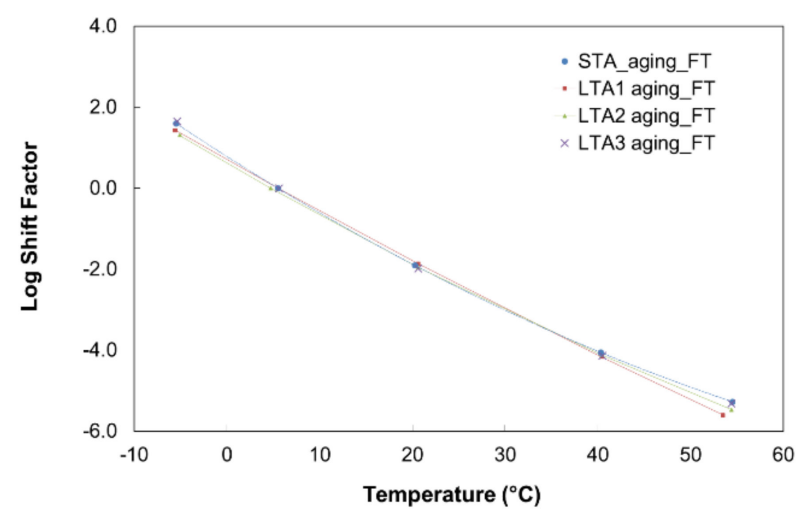

(d)

Figure 11. Dynamic modulus test results of WMA after moisture damage for each stage of aging: (a) dynamic modulus master curve (semi-log scale), (b) dynamic modulus master curve (log-log scale), (c) phase angle master curve, and (d) shift factor.

Figure 12 shows the changes in dynamic modulus of HMA and WMA mixtures at $20{ }^{\circ} \mathrm{C}$ before and after moisture damage for each stage of aging. In this study, $20{ }^{\circ} \mathrm{C}$ was chosen as the standard temperature to predict the fatigue cracking cycles using elastic modulus for each condition in the fatigue crack propagation predictive model. Figure $12 \mathrm{~b}$ shows the rate of increase according to the LTA stage based on STA, and the WMA mixture shows a sharp increase in the elastic modulus with aging compared to the HMA mixture. WMA mixture indicates that the modulus of elasticity increases by approximately $20-50 \%$ with aging, whereas the rate of increase is $10-20 \%$ compared to the HMA mixture indicating that WMA is more sensitive to aging than HMA. While the WMA mixture showed a higher elastic modulus with aging compared to the HMA mixture, the overall elastic modulus was lower in the WMA mixture than in the HMA mixture.

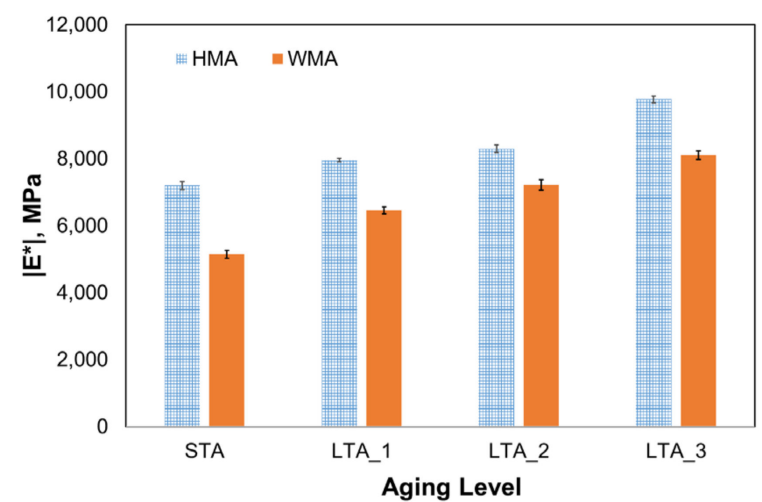

(a)

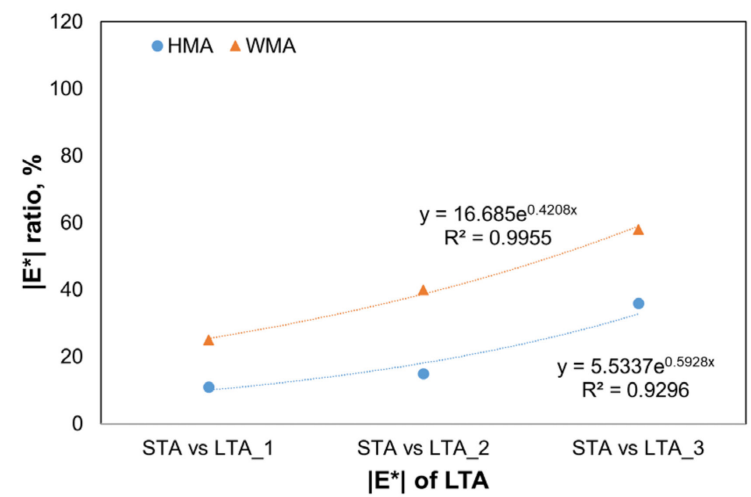

(b)

Figure 12. Dynamic modulus of HMA/WMA mixtures for each stage of aging at $20^{\circ} \mathrm{C}$ (before moisture damage): (a) dynamic modulus and (b) rate of increase.

Figure 13 shows the change in dynamic modulus after moisture damage for each stage of aging. The dynamic modulus of the WMA mixture increases rapidly with aging, as shown in Figure 13b. This indicates a rapid decrease in the dynamic modulus following moisture damage after STA, which was the initial stage of aging, suggesting that the WMA mixture exhibits an abrupt change in physical properties due to the initial moisture in use. In the WMA mixture, elastic modulus increases with aging exhibiting a similar pattern after moisture damage. This indicates that moisture damage decreases as aging continues. Thus, WMA exhibits a lower elastic modulus compared to HMA, indicating a reduced service life. 


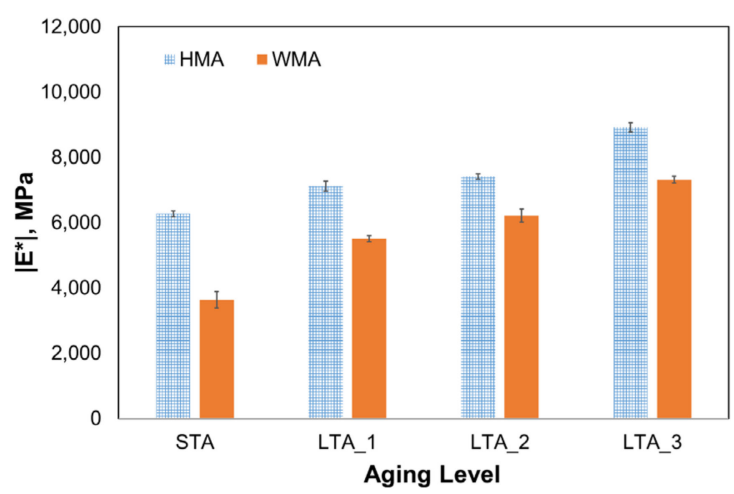

(a)

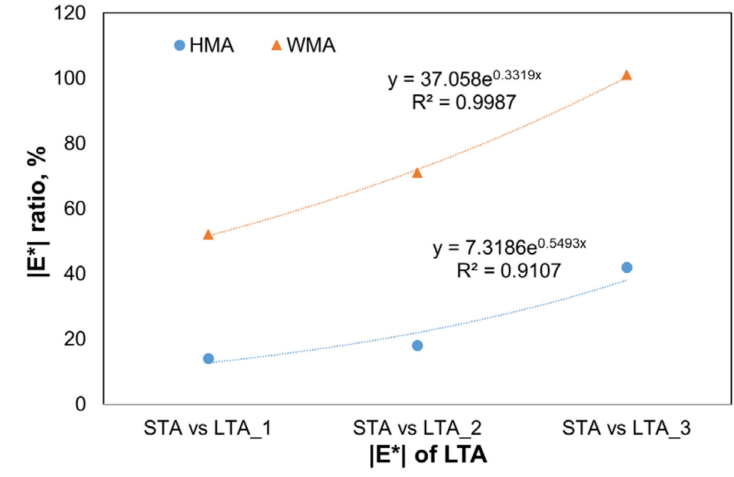

(b)

Figure 13. Dynamic modulus of the HMA/WMA mixtures for each stage of aging at $20^{\circ} \mathrm{C}$ (after moisture damage): (a) dynamic modulus and (b) rate of increase.

\subsection{Fatigue Cracking Resistance Test Results of Asphalt Mixtures}

As mentioned in Section 3.4, the fatigue cracking resistance of each mixture was evaluated controlled CX test, and the experiments were conducted by controlling the strain. In the CX test, a constant velocity strain was repeatedly applied to the specimen using the crosshead of the equipment. The test was performed by applying a tensile force to the specimen until failure at $20^{\circ} \mathrm{C}$ for a period of $10 \mathrm{~Hz}$ (haversine loading). In this study, the method proposed by Reese (1997) is used to determine the fracture time as the time when the phase angle changes rapidly [23]. After the load was applied for a certain period of time, the phase angle rapidly decreased. This was referred to as the time of failure of the specimen.

Table 5 shows the fatigue cracking performance results of HMA and WMA mixtures for each stage of aging, and Figure 14 shows the fatigue cracking performance results of the HMA and WMA mixtures due to aging. By changing the deformation size of the crosshead of the equipment to high, medium, and low levels for each stage of aging, the time at which a rapid change in the phase angle and dynamic modulus of the mixture occurred was regarded as a failure, and the number of failures at that time was determined as $\mathrm{N}_{\mathrm{f}}$. In both the mixtures, the stiffness (stress) increased with aging; however, the HMA mixture showed a decrease in the fatigue cracking cycles as the stiffness increased while the WMA mixture showed an increase in stiffness with aging and a decrease in the fatigue cracking cycles. It was also observed that the fatigue properties of the WMA mixture changed significantly with aging. In particular, the WMA mixture showed relatively low resistance to fatigue in the early stage of aging, and fatigue crack resistance gradually increased with aging. However, the fatigue cracking cycles suggested that the WMA mixture had a lower fatigue crack resistance than that of the HMA mixture under the same conditions.

Table 5. Fatigue cracking performance test results according to the aging of HMA and WMA.

\begin{tabular}{ccccc}
\hline Mix ID & No. & $\begin{array}{c}\text { Tension Stress Amplitude } \\
(\mathbf{k P a})\end{array}$ & $\begin{array}{c}\text { Peak-to-Peak Strain } \\
\text { (Microstrain) }\end{array}$ & Cycle at Failure \\
\hline \multirow{3}{*}{ HMA STA } & 1 & 987 & 78 & 52,423 \\
& 2 & 1222 & 151 & 20,423 \\
HMA LTA_1 & 3 & 1427 & 270 & 5975 \\
& 1 & 877 & 71 & 43,442 \\
HMA LTA_2 & 2 & 1127 & 148 & 15,373 \\
& 1 & 1348 & 244 & 5269 \\
HMA LTA_3 & 2 & 882 & 72 & 37,468 \\
& 3 & 1172 & 152 & 9394 \\
& 1 & 1488 & 258 & 4430 \\
& 2 & 818 & 70 & 28,487 \\
\end{tabular}


Table 5. Cont.

\begin{tabular}{ccccc}
\hline \multirow{2}{*}{ Mix ID } & No. & $\begin{array}{c}\text { Tension Stress Amplitude } \\
(\mathbf{k P a})\end{array}$ & $\begin{array}{c}\text { Peak-to-Peak Strain } \\
\text { (Microstrain) }\end{array}$ & Cycle at Failure \\
\hline \multirow{2}{*}{ WMA STA } & 1 & 788 & 104 & 10,624 \\
& 2 & 1124 & 229 & 3512 \\
WMA LTA_1 & 3 & 1561 & 371 & 2016 \\
& 1 & 896 & 79 & 16,624 \\
WMA LTA_2 & 2 & 1075 & 126 & 8624 \\
& 1 & 1530 & 355 & 224 \\
& 3 & 949 & 84 & 17,868 \\
WMA LTA_3 & 1 & 1332 & 182 & 8774 \\
& 2 & 1527 & 344 & 2563 \\
\end{tabular}

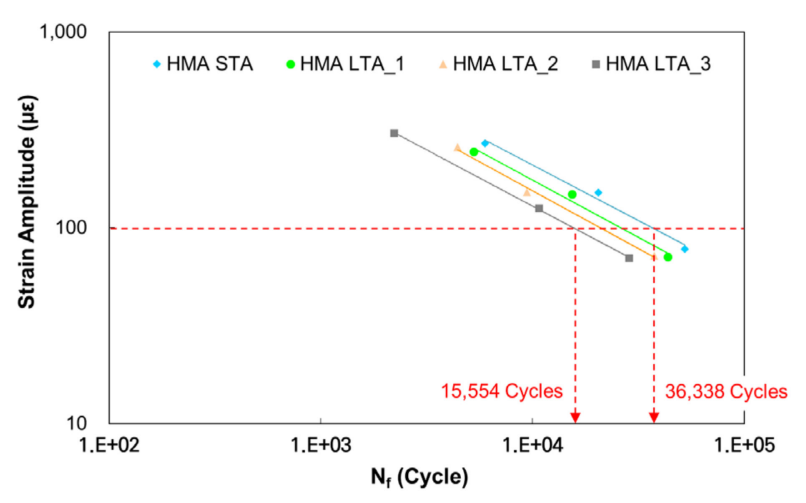

(a)

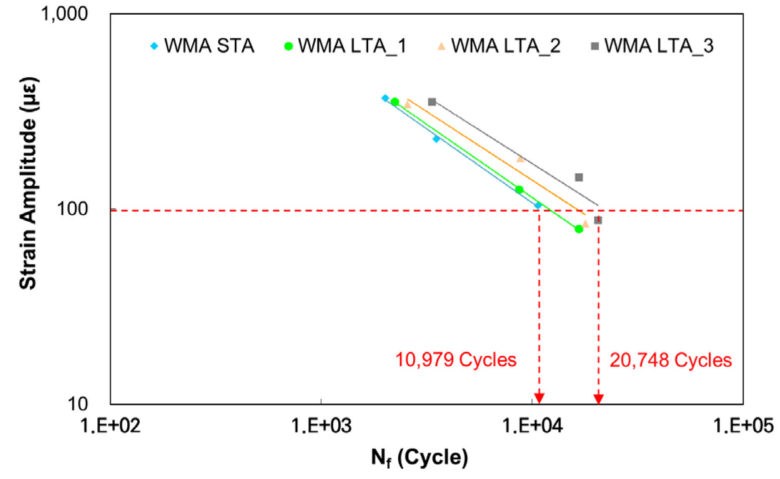

(b)

Figure 14. Allowable fatigue cracking cycles before moisture damage for each stage of aging: (a) HMA and (b) WMA.

The fatigue cracking cycles until failure can be predicted using the relationship between initial stress and strain based on the fatigue test results shown in Table 5. It was observed that the experimental coefficient value under the aging conditions can be obtained based on the experimental results. Table 6 shows the coefficient values determined under the aging conditions for each mixture through regression analysis using the elastic modulus based on the experimental results.

Table 6. Fatigue cracking prediction model coefficient of the HMA and WMA mixture for different stages of aging.

\begin{tabular}{cccc}
\hline \multirow{2}{*}{ Mix } & \multicolumn{3}{c}{ Coefficients } \\
\cline { 2 - 4 } & $\boldsymbol{k}_{\mathbf{1}}$ & $\boldsymbol{k}_{\mathbf{2}}$ & $\boldsymbol{k}_{\mathbf{3}}$ \\
\hline HMA_STA & $3.71 \times 10^{2}$ & 1.74 & 1.28 \\
HMA_LTA1 & $4.89 \times 10^{2}$ & 1.69 & 1.28 \\
HMA_LTA2 & $4.62 \times 10^{2}$ & 1.68 & 1.28 \\
HMA_LTA3 & $2.05 \times 10^{2}$ & 1.74 & 1.28 \\
WMA_STA & $3.21 \times 10^{3}$ & 1.32 & 1.28 \\
WMA_LTA1 & $4.50 \times 10^{3}$ & 1.33 & 1.28 \\
WMA_LTA2 & $5.34 \times 10^{3}$ & 1.36 & 1.28 \\
WMA_LTA3 & $8.49 \times 10^{3}$ & 1.36 & \\
\hline
\end{tabular}

Table 7 and Figure 15 show the fatigue cracking performance results of HMA and WMA mixtures due to moisture damage after aging. As shown in Table 7, the fatigue cracking resistance of HMA and WMA mixtures reduces significantly compared to that before moisture damage and further reduces at low strain. Kim (2010) evaluated the fatigue 
cracking resistance of each mixture according to moisture damage on HMA and WMA specimens collected in the field and reported that the HMA specimen showed fatigue cracking resistance of approximately $50 \%$ on an average and that of WMA specimen was approximately $20 \%$ or less [25]. In this study, both the mixtures showed similar behavioral characteristics with aging and after moisture damage. In particular, the WMA mixture showed similar behavior after moisture damage, with its fatigue cracking resistance slightly increasing as aging progressed. However, WMA generally showed lower fatigue cracking resistance than HMA.

Table 7. Fatigue cracking performance results of HMA and WMA due to moisture damage for each stage of aging.

\begin{tabular}{|c|c|c|c|c|}
\hline Mix ID & No. & $\begin{array}{c}\text { Tension Stress Amplitude } \\
(\mathbf{k P a})\end{array}$ & $\begin{array}{l}\text { Peak-to-Peak Strain } \\
\text { (Microstrain) }\end{array}$ & Cycle at Failure \\
\hline \multirow{3}{*}{ HMA STA_FT } & 1 & 1141 & 151 & 6597 \\
\hline & 2 & 1384 & 372 & 1312 \\
\hline & 3 & - & - & - \\
\hline \multirow{3}{*}{ HMA LTA_1_FT } & 1 & 924 & 81 & 14,284 \\
\hline & 2 & 1090 & 135 & 6448 \\
\hline & 3 & 1168 & 307 & 1323 \\
\hline \multirow{3}{*}{ HMA LTA_2_FT } & 1 & 945 & 83 & 11,437 \\
\hline & 2 & 1145 & 160 & 3483 \\
\hline & 3 & 1309 & 308 & 1016 \\
\hline \multirow{3}{*}{ HMA LTA_3_FT } & 1 & - & - & - \\
\hline & 2 & 1076 & 77 & 10,530 \\
\hline & 3 & 1289 & 265 & 1016 \\
\hline \multirow{3}{*}{ WMA STA_FT } & 1 & 788 & 101 & 3942 \\
\hline & 2 & 913 & 145 & 1844 \\
\hline & 3 & 971 & 258 & 573 \\
\hline \multirow{4}{*}{ WMA LTA_1_FT } & 1 & 722 & 89 & 6193 \\
\hline & 2 & 879 & 118 & 3914 \\
\hline & 3 & 983 & 254 & 788 \\
\hline & 1 & 742 & 82 & 8724 \\
\hline \multirow[t]{2}{*}{ WMA LTA_2_FT } & 2 & 874 & 121 & 4354 \\
\hline & 3 & 999 & 185 & 1837 \\
\hline \multirow{3}{*}{ WMA LTA_3_FT } & 1 & 879 & 78 & 12,914 \\
\hline & 2 & 1041 & 104 & 5952 \\
\hline & 3 & 1118 & 218 & 1688 \\
\hline
\end{tabular}

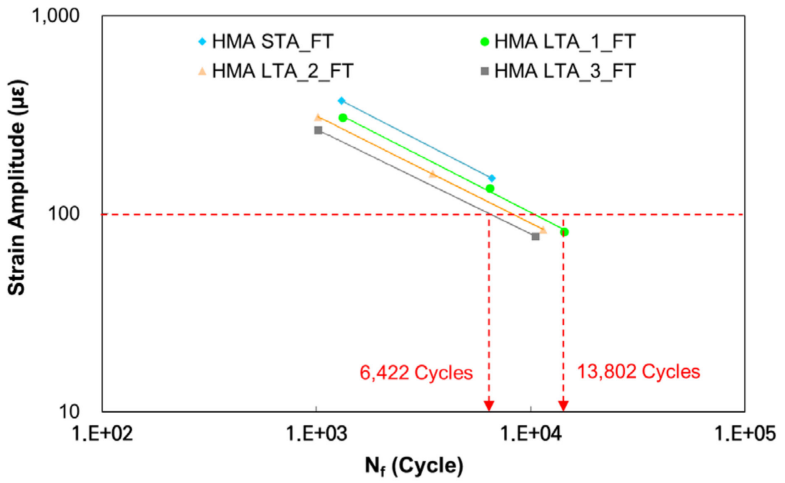

(a)

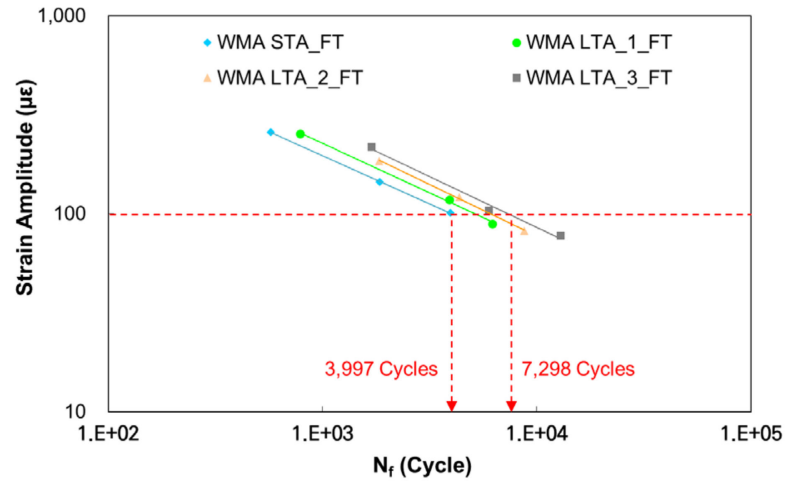

(b)

Figure 15. Allowable fatigue cracking cycles after moisture damage for each stage of aging: (a) HMA and (b) WMA.

Table 8 shows the coefficient values of the fatigue cracking resistance test according to the moisture damage of each mixture for the bottom-up cracking prediction equation mentioned above. 
Table 8. Fatigue cracking prediction model coefficients of HMA and WMA mixtures after moisture damage for each stage of aging.

\begin{tabular}{cccc}
\hline \multirow{2}{*}{ Mix } & \multicolumn{3}{c}{ Coefficients } \\
\cline { 2 - 4 } & $\boldsymbol{k}_{\mathbf{1}}$ & $\boldsymbol{k}_{\mathbf{2}}$ & $\boldsymbol{k}_{\mathbf{3}}$ \\
\hline HMA_STA_FT & $7.04 \times 10^{1}$ & 1.80 & 1.28 \\
HMA_LTA1_FT & $6.03 \times 10^{1}$ & 1.80 & 1.28 \\
HMA_LTA2_FT & $3.39 \times 10^{1}$ & 1.85 & 1.28 \\
HMA_LTA3_FT & $1.89 \times 10^{1}$ & 1.90 & 1.28 \\
WMA_STA_FT & $8.94 \times 10^{-1}$ & 2.05 & 1.28 \\
WMA_LTA1_FT & 3.61 & 1.99 & 1.28 \\
WMA_LTA2_FT & 9.78 & 1.92 & 1.28 \\
WMA_LTA3_FT & $1.42 \times 10^{1}$ & 1.92 & 1.28 \\
\hline
\end{tabular}

In the WMA mixture, the allowable loads for fatigue cracking increase with aging, unlike in the HMA mixture, which depends on the WMA additive used as well as the initial STA temperature and time. Bhaskar Pratim Das et al. (2020) statistically evaluated fatigue crack resistance according to LTA and STA for HMA and WMA mixtures and reported that the fatigue crack resistance of the WMA mixture was higher than that of HMA after LTA with the cumulative fatigue cracking cycles increasing with aging [26]. In addition, the WMA additive used in this study is a type of wax that does not react chemically owing to heat aging with no change in its physical properties following aging. In other words, waxes are oxidized because of aging caused by chemical reactions, such as those induced by UV rays rather than heat. In this study, the heat requirement for the aging condition of the additive was not met without causing the aging of the additive. Therefore, the WMA additive mixed in the asphalt binder impacted fatigue cracking resistance by maintaining the original state without aging. In the future, it is important to clearly analyze the effects of various WMA additives.

\subsection{Analysis of Fatigue Effects Due to Aging and Moisture Damage}

The concept of fatigue damage was used to determine the effect of aging and moisture damage on the fatigue damage of HMA and WMA mixtures due to aging. The lifespan was predicted based on the change in elastic modulus and tensile strain of the asphalt mixtures as well as the fatigue cracking model equation, and the degree of fatigue cracking damage was determined based on the predicted lifespan. The fatigue life $\left(N_{f}\right)$ of the asphalt pavement was evaluated by assessing the fatigue cracking performance based on the elastic modulus and coefficient determined through the fatigue cracking resistance test. The damage (D) occurring in the asphalt pavement can be defined by Equation (5) based on Miner's rule.

$$
\mathrm{D}=\frac{N_{f i}}{N_{f}}
$$

where $\mathrm{D}$ is the fatigue cracking damage, $N_{f}$ is the baseline fatigue cracking cycles of asphalt pavement, and $N_{f i}$ is the fatigue cracking cycles of asphalt pavement with aging and moisture damage.

In this study, the fatigue cracking cycles for each condition were determined using the coefficient values selected from the previously selected prediction model for the allowable fatigue cracking cycles. Figure 16 shows the fatigue effect of the HMA mixture before and after moisture damage as a function of aging time. As mentioned above, the analysis of the WMA mixture before and after moisture damage was excluded from this study because of its behavior that is different from that of the HMA mixture according to aging time. As a result of analyzing the effect of fatigue damage on HMA mixture under STA conditions, the fatigue life gradually decreased with aging that reduced by approximately $40 \%$ after 10-15 years of service life. However, the fatigue life was rapidly reduced after moisture damage during the first stage of LTA, which was $4-5$ years of service life, showing a 
reduction of $70 \%$ compared to the $100 \%$ fatigue life in the initial stage. As the service period progressed, the fatigue life due to aging gradually increased while the change in fatigue life due to moisture damage gradually decreased. Although moisture damage had a dominant influence on fatigue life in general, the effect of aging gradually increased as the service period progressed. In the first stage of LTA ( $4-5$ years), the factors affecting the fatigue life were associated with aging by approximately $20 \%$ and moisture damage by approximately $55 \%$; in the second stage of LTA (7-8 years), aging accounted for approximately $25 \%$, and moisture damage accounted for approximately $50 \%$; in the third stage of LTA (10 years or longer), aging and moisture damage accounted for approximately $40 \%$. Thus, it was observed that the effect of aging was more significant than the effect of moisture damage as the service period progressed.

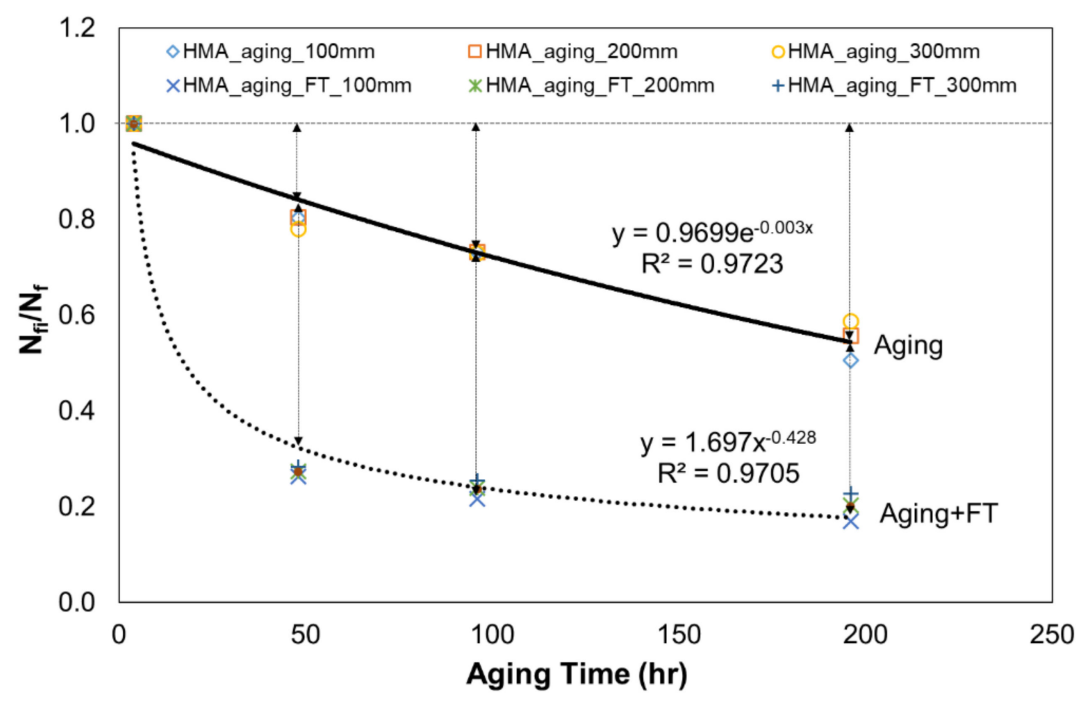

Figure 16. Analysis of fatigue effects after moisture damage for each stage of aging according to service period (HMA).

\section{Conclusions}

This study analyzed the effect of aging on resistance to fatigue cracking of asphalt mixtures due to moisture damage and aging. Additionally, the changes in material properties according to the indoor aging process and moisture damage were evaluated for HMA and WMA mixtures prepared with the same grade of asphalt binder at different initial production and construction temperatures. The dynamic modulus and change in the elastic modulus for each mixture were calculated accordingly. To evaluate the effect of aging and moisture damage on fatigue cracking, the experimental coefficient values of the predictive model were calculated for each condition.

- The elastic modulus of HMA and WMA mixtures increased with aging. For the HMA mixture, elastic modulus increased by approximately $35 \%$ as the aging time progressed from the initial stage, whereas that of the WMA mixture increased by approximately $50 \%$, suggesting that the WMA mixture has a higher sensitivity to aging according to time and temperature;

- The change in elastic modulus induced by moisture damage after aging was analyzed for both the mixtures. It was observed that the value of elastic modulus significantly reduced compared to that before moisture damage. In particular, in the WMA mixture, the initial modulus of elasticity was reduced by approximately $50 \%$, owing to moisture damage. This was because of the initial aging time and temperature considering the nature of the WMA mixture, which required a certain period of time (approximately $48 \mathrm{~h}$ ) to develop a certain stiffness (elastic modulus). The experimental coefficient values $\left(k_{1}, k_{2}\right.$, and $\left.k_{3}\right)$ of the fatigue model to predict the fatigue cracking cycles caused by aging and moisture damage after aging of HMA and WMA mixtures were 
calculated and presented for each condition. Additionally, the prediction of fatigue cracking cycles due to aging and moisture damage after aging of HMA and WMA mixtures was conducted through indoor experiments;

- Based on fatigue life prediction results using the coefficient values and tensile strain for each condition through the fatigue test, the fatigue life of the HMA mixture decreased with aging, whereas the fatigue life of the WMA mixture increased with aging;

- The fatigue lives of both the mixtures decreased rapidly after the moisture damage. The fatigue life of the HMA mixture was reduced by $60-70 \%$, and that of the WMA mixture was reduced by $80 \%$ or more after moisture damage compared to that before moisture damage. Moisture damage had a greater effect on the change in fatigue life than the aging properties;

- In the HMA mixture, the fatigue life before and after moisture damage decreased as aging progressed, indicating that the effect of aging gradually increased with an increase in the service period. As the service period progressed, it was more vulnerable to fatigue damage caused by moisture such as potholes;

- In this study, short-term and long-term aging were carried out step-by-step, after which it was subjected to water treatment. By this, the moisture damage of the asphalt mixture while it aged was simulated through water treatment. Unlike the water treatment level suggested by AASHTO T 283, the specimen was subjected to water damage in advance while short-term and long-term aging were performed to evaluate the aging and moisture damage according to the common years of packaging. The results of this evaluation revealed that moisture damage in the aged packaging can rapidly shorten its life and cause significant damage.

In this study, the material properties of the HMA mixture varied with aging and moisture damage, and the service life of the pavement was reduced by approximately $40-80 \%$ on average. In particular, the initial service life was reduced by approximately $70 \%$ owing to moisture, while the effect of aging on fatigue life increased with an increase in the service life. After 10 years, the service life of the pavement gradually decreased, and fatigue life was reduced by aging. Nevertheless, in the WMA mixture, differences in material properties and performance were recorded during its service period depending on the initial application temperature despite the use of the same grade asphalt binder. In the future, additional research is necessary to optimize the performance based on the difference in the initial application temperature.

Author Contributions: Conceptualization, S.L.Y. and H.B.P.; methodology, S.L.Y.; validation, S.L.Y., C.B., and H.B.P.; formal analysis, S.L.Y.; investigation, H.B.P.; writing—original draft preparation, S.L.Y.; writing - review and editing, H.B.P.; supervision, H.B.P.; project administration, S.L.Y.; funding acquisition, C.B. All authors have read and agreed to the published version of the manuscript.

Funding: This work was supported by the Korea Agency for Infrastructure Technology Advancement (KAIA) grant funded by the Ministry of Land, Infrastructure, and Transport (Grant No. 21POQW-B152342-03).

Data Availability Statement: Not applicable.

Conflicts of Interest: The authors declare no conflict of interest.

\section{References}

1. Little, D.N.; Jones, D.R. Chemical and Mechanical Processes of Moisture Damage in Hot-Mix Asphalt Pavements. In Proceedings of the Moisture Sensitivity of Asphalt Pavements National Seminar, San Diego, CA, USA, 4-6 February 2003.

2. Hicks, R.G.; Santucci, L.; Aschenbrener, T. Introduction and seminar objectives. In Proceedings of the Moisture Sensitivity of Asphalt Pavements National Seminar, San Diego, CA, USA, 4-6 February 2003.

3. He, J.; Liu, L.; Yang, H.; Aliha, M.R.M.; Karimi, H.R. Contribution of interface fracture mechanism on fracture propagation trajectory of heterogeneous asphalt composites. Appl. Sci. 2021, 11, 3013. [CrossRef]

4. Yang, D.; Karimi, H.R.; Aliha, M.R.M. Comparison of testing method effects on cracking resistance of asphalt concrete mixtures. Appl. Sci. 2021, 11, 5094. [CrossRef] 
5. Shaker, S.; Aliha, M.R.M.; Keymanesh, M.R. Aging effect on combined mode fracture resistance of bitumen. Fatigue Fract. Eng. Mater. Struct. 2019, 42, 1609-1621. [CrossRef]

6. Zhu, J.; Ma, T.; Fan, J.; Fang, Z.; Chen, T.; Zho, Y. Experimental study of high modulus asphalt mixture containing reclaimed asphalt pavement. J. Clean. Prod. 2020, 263, 121447. [CrossRef]

7. Zhou, Y.; Hou, D.; Manzano, H.; Orozco, C.A.; Geng, G.; Monteiro, P.J.M.; Liu, J. Interfacial connection mechanisms in calciumsilicate-hydrates/polymer nanocomposites: A molecular dynamics study. ACS Appl. Mater. Interfaces 2017, 9, 41014-41025. [CrossRef]

8. Brown, S.F.; Scholz, T.V. Development of laboratory protocols for the aging of asphalt mixtures. In Proceedings of the 2nd Eurasphalt and Eurobitume Congress, Barcelona, Spain, 20-22 September 2000.

9. AASHTO. Standard Method of Test for Resistance of Compacted Asphalt Mixtures to Moisture-Induced Damage; American Association of State Highway and Transportation Officials: Washington, DC, USA, 2014.

10. Lee, J.; Moon, S.J.; Im, J.; Yang, S. Evaluation of moisture susceptibility of asphalt mixtures using dynamic modulus. J. Test. Eval. 2016, 45, 1280-1288. [CrossRef]

11. Saad, I.; Sarsam, I.; Lutfi, A.-Z. Resistance to moisture damage of recycled asphalt concrete pavement. J. Eng. 2015, $21,45-54$.

12. Das, P.K.; Birgisson, B.; Jelagin, D.; Kringos, N. Investigation of the asphalt mixture morphology influence on its ageing susceptibility. Mater. Struct. 2015, 48, 987-1000. [CrossRef]

13. Seoul Institute of Technology. Maintenance Methods Depending on Pavement Deterioration Types; SIT: Seoul, Korea, 2020.

14. Seoul Metropolitan Government. Available online: https:/ / opengov.seoul.go.kr/press/18456873 (accessed on 30 October 2021).

15. Ministry of Land, Infrastructure and Transport. Asphalt Mixture Production and Construction Guidelines; MOLIT: Sejong City, Korea, 2017.

16. Dougan, C.E.; Stephens, J.E.; Mahoney, J.; Hansen, G. E*-Dynamic Modulus Test Protocol—Problems and Solutions; Federal Highway Administration: Washington, DC, USA, 2003.

17. Baek, C.M. Investigation of Top-Down Cracking Mechanisms Using the Viscoelastic Continuum Damage Finite Element Program. Ph.D. Thesis, North Carolina University, Chapel Hill, NC, USA, 2010.

18. Bell, C.A.; Wieder, A.J.; Fellin, M.J. Laboratory Aging of Asphalt-Aggregate Mixtures: Field Validation; Strategic Highway Research Program: Washington, DC, USA, 1994.

19. Kim, Y.R.; Hintz, C.; Rad, F.Y.; Elwardany, M.; Underwood, S. Long-Term Aging of Asphalt Mixtures for Performance Testing and Prediction; National Cooperative Highway Research Program: Washington, DC, USA, 2015.

20. AASHTO. Standard Practice for Mixture Conditioning of Hot Mix Asphalt; American Association of State Highway and Transportation Officials: Washington, DC, USA, 2002.

21. Bonaquest, R. Mix Design Practices for Warm Mix Asphalt; National Cooperative Highway Research Program: Washington, DC, USA, 2009.

22. AASHTO. Method of Test for Determining Dynamic Modulus of Hot Mix Asphalt (HMA); Association of State Highway and Transportation Officials: Washington, DC, USA, 2007.

23. Reese, R. Properties of aged asphalt binder related to asphalt concrete fatigue life. J. Assoc. Asph. Paving Technol. 1997, 66, 604-632.

24. Safaeia, F.; Lee, J.; do Nascimento, L.A.H.; Hintz, C.; Kim, Y.R. Implications of warm-mix asphalt on long-term oxidative ageing and fatigue performance of asphalt binders and mixtures. Road Mater. Pavement Des. 2014, 15, 45-61. [CrossRef]

25. Kim, Y.R.; Baek, C.M. Evaluation of moisture susceptibility in a warm mix asphalt pavement: US 157, Hurdle Mills, NC. In Proceedings of the 2nd International Warm-Mix Conference, St. Louis, MI, USA, 13 October 2011.

26. Das, B.P.; Das, S.; Siddagangaiah, A.K. Probabilistic modeling of fatigue damage in asphalt mixture. Constr. Build. Mater. 2020, 269, 121300. [CrossRef] 